\title{
Mujeres indígenas y múltiples formas de discriminación en el trabajo doméstico
}

\section{Indigenous women and multiple forms of discrimination in domestic work}

\author{
Belinda García ${ }^{a}$
}

\section{Resumen}

Las mujeres indígenas jóvenes en su mayoría son las que obtienen empleo como trabajadoras domésticas. Si bien las mujeres indígenas son discriminadas por la legislación, en general el pago es el $60 \%$ del salario mínimo legal para cualquier trabajadora doméstica, pero en el caso de las mujeres indígenas incluso reciben pagos en forma diferenciada por debajo del $60 \%$ o con especias. La discriminación que sufren tiene diferentes aristas, por ser mujeres como condición inferior a un hombre, por ser originarias o pertenecientes a descendencia originaria, por los rasgos físicos, el hecho de hablar solo el idioma nativo o guaraní paraguayo y no el español; también es un motivo de discriminación hasta por la vestimenta típica o casual que utilizan, donde los lugares de trabajo las obligan a cambiarse y utilizar una determinada vestimenta despojándolas de su cultura originaria.

Palabras clave: mujer indígena, trabajo doméstico, discriminación, género.

Kera Yvoty: reflexiones sobre la cuestión social. Vol. 1, 2016, $111-117$.

ISSN (impreso): 2519-7797

a Universidad Nacional de Asunción, Instituto de Trabajo social, Paraguay.

Correspondencia a: beligarciapy@gmail.com

Cita:

García, B. (2016). Mujeres indígenas y múltiples formas de discriminación en el trabajo doméstico. Kera Yvoty: reflexiones sobre la cuestión social, 1, 111-117.

Recibido:

1 noviembre 2016

Aceptado:

18 diciembre 2016

\begin{abstract}
Abstrac
Young native women are mostly employed as domestic workers. Native women are discriminated against by legislation, while in general the payment is $60 \%$ of the legal minimum wage for any domestic worker, in the case of native women they receive payments in a differentiated form below $60 \%$ or with spices.

The discrimination they suffer has different edges, because they are women as a condition inferior to a man, because they are native or belonging to original offspring, because of their physical features, and speaking only the native language or Paraguayan Guarani but not Spanish; in addition the typical or casual clothing they use is also a reason for discrimination since workplaces force them to change and use a certain clothing stripping them of their original culture.
\end{abstract}

Keywords: native women, domestic work, discrimination, gender. 


\section{Introducción}

El trabajo doméstico es uno de los trabajos más invisibles dentro de la división social del trabajo y ha sido un trabajo impuesto socialmente a las mujeres en sus hogares.

Esta cuestión social necesita ser analizada desde una perspectiva histórica y socioeconómica, dentro de la dinámica de la reproducción de la fuerza de trabajo en el sistema capitalista de producción.

A su vez es necesario comprender la explotación de la fuerza de trabajo femenina con la cuestión de clase subalterna y género en relación al patriarcado que considera a la mujer como un objeto de transferencia económica y sustento de la vida del proletario, como sirvienta y reproductora.

$\mathrm{Si}$ bien existen múltiples investigaciones sobre mujer y trabajo doméstico, no abordan específicamente el trabajo doméstico de mujeres indígenas, no existen trabajos que nos den a conocer datos que nos demuestren niveles de invisibilidad de las mujeres trabajadoras, ya que en el proceso de revisión bibliográfica se encuentran pocos antecedentes a nivel nacional en registro y sistematización de la cuestión social en relación a la mujer indígena trabajadora doméstica.

En cuanto a la discriminación existen también trabajos que muestran índices de discriminación en distintos ámbitos. En lo laboral, no se aborda la cuestión social mencionada.

La categorización del trabajo doméstico como una cuestión social representa una manifestación de la dinámica social y la reproducción de súper explotación de la fuerza de trabajo de mujeres en la incorporación al mercado laboral, donde las mujeres ofrecen una fuerza de trabajo de menor costo, para quienes compran su fuerza de trabajo.

\section{Objetivo}

Este trabajo tiene por objetivo general caracterizar la discriminación múltiple en mujeres indígenas trabajadoras domésticas.
Los objetivos específicos son:

-Conocer el nivel de invisibilidad

y discriminación de las mujeres indígenas en el trabajo doméstico.

-Identificar los procesos de resistencia de las mujeres indígenas que realizan tareas domésticas.

\section{Metodología}

El estudio de la cuestión social de discriminación en el trabajo doméstico en mujeres indígenas es de tipo exploratorio, se basa en revisiones bibliográficas que recogen tres entrevistas a informantes pertenecientes de pueblos originarios y fuentes secundarias para el análisis cualitativo.

Se intenta conocer la inserción de la mujer indígena en el trabajo doméstico así como las condiciones laborales en que se desarrollan y la situación de vulnerabilidad en cuando a la discriminación en varios ámbitos.

Las técnicas utilizadas en el proceso de la investigación de campo fueron la observación y entrevista estructurada de carácter formal con anticipación previa a los/as entrevistados/as. Se aplicaron 3 entrevistas semiestructuradas obteniendo datos de primera fuente, con entrevistas claves de los pueblos Qom, Guaraní Occidental y Lengua Maskoy.

En el análisis de los datos se vinculan y analizan categorías teóricas que se plantean en el marco teórico referencial en relación a los datos que se obtuvieron en la recolección de información.

La revisión de la bibliografía se realizó a partir del análisis de los contenidos en base al área temática adjuntando las categorías generales de la cuestión social.

\section{Resultados}

\subsection{Cuestión Social y proletarización de indígenas \\ Para entender la llamada cuestión social desde su contexto histórico como producto de las desigualdades y}


contradicciones del sistema capitalista es necesario entender la categoría desde dinámica del proceso histórico (Martinelli, 1992). La autora nos presenta vertientes desde donde parte la diversidad de compresión del capitalismo como categoría histórica atendiendo su propia complejidad, encontrando un rigor teórico sobre el pensamiento de Karl Marx donde se explican las condiciones del proceso de producción del trabajo social donde se evidencia, que el capital es una relación social y el capitalismo un sistema de producción definiendo una estructura social asentada sobre la propiedad privada de los medios de producción asociado a una estructura de ideas y fases en la historia.

Laalienación de los trabajadores tiene que ver con su mismo desconocimiento del papel que interpretan en el proceso total de producción social. El crecimiento de las ciudades industriales fue una consecuencia directa de la expansión del capital y sus exigencias dibujando aún más la polarización de las clases entre proletarios y burgueses, donde la desvalorización del ser humano (Martinelli, 1992) se convirtió en ley del capital y paso a valorizarse la mercancía,

La naturaleza no produce, por un lado, poseedores de dinero o de mercancías y por otro, meros poseedores de las propias fuerzas de trabajo. Esta relación no tiene origen en la naturaleza, ni es incluso una relación social común a todos los periodos históricos. (Marx, 1984)

La relación social creada dentro del proceso de trabajo también es parte de un proceso de rebeldía y resistencia (Iamamoto, 2003) porque involucran a los trabajadores y trabajadoras siendo ellos y ellas las que generan una resistencia a la desigualdad estando dentro del sistema de producción capitalista y se recrean distintas expresiones que postulan que es posible la construcción de otras relaciones.

Sin duda la raíz de la desigualdad social hasta nuestros días corresponde al problema de la tierra, siendo un país con la mayor desigualdad de la región, el latifundio se arrastra ya desde la época independiente.

Dentro de ese contexto se dio la proletarización de los pueblos indígenas y de campesinos como requerimiento capitalista en el proceso de liberalización de la economía paraguaya. El capital constante era entregado para la producción de materias primas, quedándose el Estado con todo excedente producido.

Con el objetivo de la invasión y destrucción de la fortalecida economía interna paraguaya que negaba el orden capitalista colonial inglés en la región, el imperialismo logro instalar en los países que participaron de la guerra cuentas de créditos, sometiendo a préstamos con libras esterlinas a todos los países que participaron de la guerra, como el posterior control económico y recuperación del mercado, donde la población paraguaya era blanco de exterminio, ubicando una desigualdad social asentada sobre la propiedad privada de la tierra, instalando el modo semi esclavista. Asi, del único estado foco de resistencia antimperialista en Latinoamérica solo quedaban cenizas (Coronel, 2011).

El Estado paraguayo realiza una regulación de la relación capital-trabajo dentro del desarrollo capitalista con la creación de empresas estatales creando las bases para el surgimiento del empresariado privado hasta estos días parte de la burguesía hegemónica del país.

\subsection{Categorización del trabajo doméstico y mujeres indígenas}

La categorización del trabajo doméstico como cuestión social que representa una manifestación de la dinámica social y la reproducción de súper explotación de la fuerza de trabajo de mujeres, en la incorporación al mercado laboral, donde las mujeres ofrecen una fuerza de trabajo de menor costo, para 
quienes compran su fuerza de trabajo.

Las mujeres son parte del proceso histórico de la mercantilización del trabajo, en donde desde el cercamiento de tierras comunes hasta la desposesión de sus cuerpos y la trasformación de éstos en instrumento de trabajo, tuvo connotaciones específicas para las mujeres (Quiroga \& Gago, 2013), estableciendo una división sexual del trabajo, donde según Federici (2004).

En la nueva organización del trabajo todas las mujeres se convirtieron en bien comunal, pues una vez que las actividades de las mujeres fueron definidas como notrabajo se establece una doble dependencia sobre el patriarcado en carácter de esclavas casi, como los inicios capitalistas en la época colonialista en América Latina. "Los empresarios históricamente han preferido contratar mujeres en ciertos mercados de trabajo" (Revelo, 2001). Esto, por el abaratamiento de su fuerza de trabajo y también por ciertos factores que determinan el rendimiento del precio de costo del salario en consideración a la producción diaria de mercancías.

Este proceso desigual se da con mayor fuerza en mujeres, en la combinación de la explotación de clase y opresión de género como un sistema unitario sobre las trabajadoras domésticas.

\subsection{Invisibilidad del trabajo doméstico de mujeres indígenas}

Una histórica invisibilidad del trabajo femenino y en especial el doméstico en el que se recrean las condiciones de funcionamiento del sistema capitalista, la atención social puesta sobre las trabajadoras domésticas en la asistencia social, de carácter a las respuestas a esta expresión de la cuestión social.

Porque la división del trabajo por género es la primera división del trabajo, y en las llamadas sociedades primitivas, es la única división del trabajo institucionalizada". (Young, 1994)
Entendiendo que las relaciones patriarcales de opresión hacia las mujeres y el proceso de mercantilización de la fuerza de trabajo, son procesos históricos distintos sobre un proceso social de funcionamiento unitario como lo es capitalismo-patriarcado cuasi de funcionamiento semiautónomo pero de interrelación directa, donde las relaciones de género no son las mismas que las relaciones de clases según Revelo (2001) abordando al patriarcado como un sistema político sobre una historicidad de dominacióny poder. Dondelas relaciones de clase y relaciones de género juntas son una relación distinta y por ende una interacción de relaciones de mayor complejidad.

Esto puede ejemplificarse a través del trabajo doméstico ubicándolo por un lado como naturalización de la producción y reproducción familiar, donde las tareas domésticas son asumidas casi "obligatoriamente por naturaleza" (Revelo, 2001) y por otro en una distinción sobre el tiempo y espacio de trabajo, sobre el salario/trabajo doméstico/trabajo/gremio sindical, etc. Siendo la mujer ejecutora de 2 a 3 jornadas laborales en distintas formas de producción diaria de trabajo dentro de la dinámica del trabajo social.

Un 8 de julio de 1884 el servicio doméstico fue reglamentado con una ordenanza. Fue el primer trabajo reglamentado pero protegiendo más al empleador, convirtiéndose en un instrumento de control sobre la población pobre (Barreto, 2012). Entonces se genera una amplia migración ya en ese entonces del campo a la ciudad para ofrecer sus servicios domésticos. No existía salario para la profesión y este se establecía de común acuerdo (Barreto, 2012).

Luego de 130 años algunas características del servicio doméstico no han cambiado.

\section{Discusión}

Según la EPH en el 2000 existía 148.628 empleados/as domesticas del cual $93 \%$ son mujeres. Actualmente las 
trabajadoras domésticas y según la EPH (2013) existe un 7,2\% de la población en un total de 3.274.819 personas de las cuales o,9 corresponden a hombres y 16,0 de mujeres que realizan el trabajo doméstico. No se observan índices cuantas de esas mujeres son indígenas.

Las mujeres indígenas son transmisoras de la cultura y cosmovisión de sus pueblos, una de las tantas características de las mujeres indígenas es la de no dejar a un lado la cuestión tradicional, en su mayor parte las mujeres se dedican a la artesanía, la crianza de las criaturas, la enseñanza del idioma y los canticos, y todo lo relacionado con la cultura.

Este trabajo lo realizan en forma general las mujeres indígenas en las dos regiones del país. Esto se observa con mayor frecuencia en el sector urbano sector en el que las mujeres cuidan a una cantidad de hijos suyos y es la encargada de realizar esa tarea, además de trabajar para el sustento de todos/as.

Aunque muchas mujeres indígenas aún permanecen en sus comunidades, muchas optan por la migración a los sectores urbanos y de periferia a buscar trabajo.

En el Chaco paraguayo existe una complejidad de situaciones. En el sector rural además de realizar todas éstas tareas las mujeres deben encargarse de la provisión de alimentos a sus hogares. En cambio, en los sectores semi urbanos las mujeres migran a ciudades en busca de oportunidades laborales.

\subsection{Discriminación de género en las leyes laborales.}

La discriminación es un hecho que marca la realidad de los pueblos originarios y de las mujeres trabajadoras, existiendo una discriminación laboral legal hacia las trabajadoras domésticas no indígenas solo con la remuneración del 6o\% del salario mínimo legal.

La discriminación de género por ser mujeres y por el hecho de ser originarias, a la par trabajadoras domésticas además consiste en graves violaciones a los derechos humanos, "como en el caso que la región del Chaco. Hay registros de mujeres nivaclé que trabajan de domesticas con los menonitas y ellas son encerradas dentro de las casas, algunas trabajan allí por 3 hasta 5 meses pudiéndose considerar un hecho de trata de mujeres" (entrevista $\mathrm{N}^{\circ}{ }_{1}, 2014$ )

La discriminación legal en cuanto al ámbito laboral, encuentra una legislación pobre e incapaz de atender la violación de derechos laborales sobre todo en lo que tiene que ver con la remuneración que en muchas ocasiones se trata de víveres, cupos para comprar en supermercados o semillas y la permanencia de las mujeres originarias en sus lugares de trabajo.

En cuanto a la remuneración ganan entre Gs. 600.000 en las zonas urbanas de Asunción y gran Asunción, con la posibilidad de vivir en donde quieran o salir de las casa los fines de semana. En comunidades menonitas del Chaco en los sectores urbanos la remuneración salarial a las mujeres se da a través de provistas, y en los sectores rurales del Chaco alcanzan a pagar de Gs.100.ooo por mes hasta Gs.2.00o.ooo por trabajar de 4 a 5 meses en una condición de encierro.

También hay registro de mujeres que indican la humillación que sufren dentro de los lugares donde trabajan como domesticas e "inclusive como se las somete sexualmente" (entrevista $\mathrm{n}^{\circ} 2$ ).

\subsection{Trabajo doméstico ligado a explotación sexual en las mujeres indígenas}

Un gran número de mujeres domesticas indígenas que trabajan con o sin retiro también son explotadas sexualmente. (entrevista $\mathrm{N}^{\circ} 1$, entrevista $\mathrm{N}^{\circ} 2$, 2014).

Dentro de la mesa indígena del Paraguay hay mujeres trabajadoras domésticas. Registran 82 mujeres indígenas que son empleadas domésticas de las comunidades Tarumandy y Cerro Poty de Asunción. Las trabajadoras domésticas son 
contratadas normalmente en el Chaco por los menonitas, en las colonias de Filadelfia, Loma Plata y Neuland. Las trabajadoras domésticas indígenas no tienen IPS, no tienen aguinaldo, no tienen jubilación no tienen ningún derecho laboral, como otras empleadas domésticas.

"Se las contrata por ser indígenas y esa es la razón por la cual les paga poco" (entrevista $\mathrm{n}^{\circ}$ 3, 2015). Hay entonces una diferencia entre una trabajadora doméstica y una trabajadora doméstica indígena: $\mathrm{Su}$ condición étnica.

Se ven obligadas a la explotación sexual como algo que roza la normalidad de su día a día, acostumbradas a que la discriminen por ser indígena y la maltraten todos los miembros del lugar donde trabaja sea en el campo o ciudad.

No existen antecedentes que demuestren políticas sociales o de programas de promoción de derechos humanos y derechos laborales que protejan las mujeres trabajadoras domésticas indígenas.

Pese a la organización de algunas trabajadoras ésta se torna muy débil y sin estructura de trabajo para el seguimiento de los casos de discriminación y abuso sexual que sufren las domesticas y otras denuncias.

\subsection{Percepción de promoción de derechos laborales de mujeres indígenas}

No se conocen de proyectos de promoción de los derechos laborales de las mujeres indígenas, y se considera que el "problema de los pueblos indígenas es total, pero que afecta más a las mujeres" (entrevista $\left.\mathrm{n}^{\circ} 1,2014\right)$.

La Secretaria de la mujer hizo un programa de la concientización de los derechos de las mujeres indígenas pero no se hace el programa de desarrollo a largo plazo para quitar de raíz eso. Como organización tenemos una postura de que la pobreza y la discriminación vienen de la sociedad paraguaya que esta infiltrada en la cultura occidental (entrevista $n^{\circ} 1,2014$ ).
En el trabajo doméstico indígena pueden observarse muchos factores que inciden en la dinámica social de las mujeres. La migración de éstas de sus comunidades, desde sector rural al urbano, tiene que ver con un problema de territorio.

Aunque sean transmisoras de las culturas de sus pueblos, también son quienes sustentan a las familias desde la realización de artesanías hasta el trabajo doméstico.

En ese sentido el trabajo doméstico, constituye una serie de condicionamientos que atacan a las mujeres que tienen que ver con la permanencia en su lugar de trabajo. Las mujeres no hablan no opinan, "no son gente".

Es necesarioelestudioen profundidad del trabajo doméstico en mujeres indígenas, y las condiciones de explotación laboral de carácter semi esclavista en el sector rural, principalmente en el Chaco.

Es necesario también entender que las resistencias diarias de las mujeres trabajadoras domésticas indígenas están enmarcadas con la persistencia en la transmisión de la cultura a sus hijos/ as, hermanos/a, pese a las condiciones laborales de explotación y violación de derechos que las afectan.

\section{Referencias Bibliográficas}

Barreto, A. M. (2012). Voces de Mujer en la historia paraguaya, 200 años después. Asunción: AIETI.

Coronel, B. (2012). Paraguay, la vanguardia capitalista del Siglo XIX. En L. Rojas (Ed.), Proceso histórico de la economía paraguaya (pp. 179-205). Asunción: Secretaría Nacional de Cultura.

DGEEC. (2014). Encuesta Permanente de Hogares. Principales Resultados. Paraguay 2013. Fenrnando de la Mora: DGEEC.

Federici, S. (2004). Autonomedia, Calibán y la bruja. Mujeres, cuerpo y acumulación originaria. Madrid: Traficantes de Sueños.

Iamamoto, M. (2003). El Servicio Social en 
la contemporaneidad. En A. Pastorini \& C. Montaño (Ed.), El Servicio Social en la contemporaneidad. Trabajo y formación profesional. San Pablo: Cortez Editora.

Karl, M. (1984). La llamada Acumulación Originaria. In El capital (cap. 24). Recuperado de https://www.marxists. org/espanol/m-e/186os/eccx86s.htm

Martinelli, M. L. (1992). Servicio social: identidad y alineación. San Pablo: Cortez Editora.

Quiroga, N. \& Gago, V. (2013). Los comunes en femenino. Cuerpo y poder ante la expropiación de las economías para la vida. Recuperado de http://dx.doi. org/10.15359/eys.19-45.1
Revelo, P. (2001). La clase y el género ¿Dos conceptos irreconciliables a finales del milenio? Notas para un debate. Nueva Antropología, 18(59), 145-16o.

Young, I. (1994). Marxismo y feminismo, más allá del "matrimonio feliz". Una crítica al sistema dual. Cielo por asalto, 2(4). Recuperado de http://www. democrasiasocialista.org/wp-content/ uploads/2014/03/13910a361-YoungMarxismo-y-feminismo.pdf 